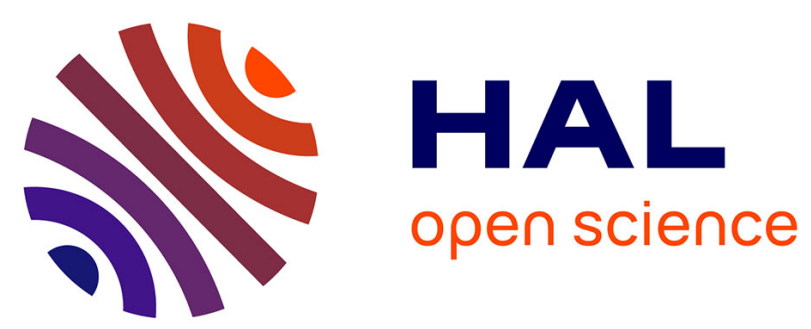

\title{
3D COLORED MESH STRUCTURE-PRESERVING FILTERING WITH ADAPTIVE P-LAPLACIAN ON DIRECTED GRAPHS
}

\author{
Sébastien Bougleux, Olivier Lézoray, Anass Nouri
}

\section{To cite this version:}

Sébastien Bougleux, Olivier Lézoray, Anass Nouri. 3D COLORED MESH STRUCTUREPRESERVING FILTERING WITH ADAPTIVE P-LAPLACIAN ON DIRECTED GRAPHS. 26th IEEE International Conference on Image Processing (ICIP 2019), Sep 2019, Taipei, Taiwan. 10.1109/ICIP.2019.8803642 . hal-02297223

\section{HAL Id: hal-02297223 \\ https://hal.science/hal-02297223}

Submitted on 25 Sep 2019

HAL is a multi-disciplinary open access archive for the deposit and dissemination of scientific research documents, whether they are published or not. The documents may come from teaching and research institutions in France or abroad, or from public or private research centers.
L'archive ouverte pluridisciplinaire HAL, est destinée au dépôt et à la diffusion de documents scientifiques de niveau recherche, publiés ou non, émanant des établissements d'enseignement et de recherche français ou étrangers, des laboratoires publics ou privés. 


\section{D COLORED MESH STRUCTURE-PRESERVING FILTERING WITH ADAPTIVE P-LAPLACIAN ON DIRECTED GRAPHS}

\author{
S. Bougleux, O. Lézoray* \\ Normandie Univ, UNICAEN, ENSICAEN \\ CNRS, GREYC, 14000 Caen, France
}

\author{
A. Nouri \\ Vision Lab. Isen Brest, L@bISEN \\ 29228 Brest Cedex, France
}

\begin{abstract}
Editing of 3D colored meshes represents a fundamental component of nowadays computer vision and computer graphics applications. In this paper, we propose a framework based on the $p$-laplacian on directed graphs for structure-preserving filtering. This relies on a novel objective function composed of a fitting term, a smoothness term with a spatially-variant $p$ TV norm, and a structure-preserving term. The last two terms can be related to formulations of the $p$-Laplacian on directed graphs. This enables to impose different forms of processing onto different graph areas for better smoothing quality.
\end{abstract}

Index Terms - 3D colored meshes, graph signal, structure preserving filtering, $p$-Laplacian.

\section{INTRODUCTION}

Structure-preserving filtering is one of the most important processing tasks dedicated to image editing and computational photography. During the last decade, many structurepreserving smoothing filters have been proposed so far [1, 2, 3, 4]. To enable the editing of an image, they decompose it into a base layer containing the rough structures with preserved edges and several layers of increasing level of details. From this hierarchical representation, image editing tasks such as smoothing, abstraction, and sharpening can be performed [5]. At the same time, the advent of low cost 3D sensors has enabled the large development of 3D scanning. Using either a real 3D scanner or a set of images obtained by moving around an object, one can easily obtain 3D colored point clouds or meshes where each point or vertex is described by its 3D position and RGB color. However, the visual quality of the acquired data is not always of sufficient especially when a further 3D printing is planned. In addition, if the 3D data has to be used in virtual reality applications, it often has to be post-processed to be visually simplified. With such 3D processing tasks to be performed, there is interest into the development, as this has been done for images, of

\footnotetext{
* This work received funding from the Agence Nationale de la Recherche (ANR-14-CE27-0001 GRAPHSIP), from the European Union FEDER/FSE 2014/2020 (GRAPHSIP project), and the support of NVIDIA Corporation with the donation of a Titan Xp GPU used for this research.
}

structure-preserving filters that operate on 3D colored data. Very few works have considered this kind of problem. Some structure-preserving filters have been extended to 3D meshes but they only consider the editing of the 3D points' coordinates to sharpen the mesh [6, 7]. Some recent works have considered the extension for 3D colored meshes by a 3D extension of unsharp masking [8] or with the use of morphological filters to obtain a hierarchical decomposition of the 3D color information [9, 10] .

In this paper we propose a structure-preserving filter based on an objective function composed of three terms (Sec. 3): a data-fitting term, a smoothness term and a structure preserving term. To have a more adaptive filtering, the smoothness term relies on a spatially-variant $p$-total variation norm $(p \mathrm{TV})$. This extends our previous works [11] that considered only the two first terms with a fixed $p$ TV norm. The smoothness term can be related to formulations of the $p$-Laplacian on directed graphs (Sec. 2). Finally the structure-preserving term enables to preserve the most important structures. This enables to impose different forms of regularization onto different $3 \mathrm{D}$ areas for better smoothing quality, as illustrated and discussed in Sec. 4.

\section{P-LAPLACIANS ON DIRECTED GRAPHS}

Graphs provide a powerful and common structure for representing and processing structured and unstructured data, like images, colored 3D point clouds or meshes. The data are assumed to be composed of elements living in $\mathbb{R}^{n_{c}}$, e.g. 3D points or RGB colors. A data of $n_{v}$ elements is represented by a real matrix $\mathrm{F}=\left[f_{i, c}\right]_{i \in N_{v}, c \in N_{c}}$, with $N_{v}=\left\{1, \ldots, n_{v}\right\}$ and $N_{c}=\left\{1, \ldots, n_{c}\right\}$, so that $f_{i, c}$ encodes the $c$-th component of the $i$-th element. Alternatively, the $i$-th element can be represented by a vector $\mathrm{f}_{i}=\left[f_{i, c}\right]_{c \in N_{c}}$. The elements are connected by edges to form a graph used as a domain for processing the data. Since connections do not usually have the same strength, a weight is associated to each edge, and the graph can be fully represented by its weighted vertex-vertex adjacency matrix $\mathrm{W}=\left[w_{i, j}\right]_{i \in N_{v}, j \in N_{v}}$, with $w_{i, j} \in(0,+\infty)$ if the elements $i$ and $j$ are connected by an edge from $i$ to $j$, or $w_{i, j}=0$ if they are not. Graphs are here assumed to 
be without self-loops $\left(w_{i, i}=0\right)$ and directed $\left(w_{i, j}\right.$ and $w_{j, i}$ can be different). This is motivated by the construction of graphs based on $k$-nearest neighbors, that are known to produce asymmetric connections in general, i.e. an edge from $i$ to $j$ does not necessarily imply an edge from $j$ to $i$. Such graphs are commonly symmetrized, i.e. $\mathrm{W}$ is replaced by $\frac{1}{2}(\mathrm{~W}+$ $\mathrm{W}^{T}$ ), before processing the data. While this is mathematically correct for most methods based on the unnormalized Laplacian $\mathrm{L}=\operatorname{diag}\left(\left(\mathrm{W}+\mathrm{W}^{T}\right) 1_{n_{v}}\right)-\left(\mathrm{W}+\mathrm{W}^{T}\right)$ [12, 13, 14], this is not the case for most second-order operators, especially non-linear operators like curvatures and $p$-Laplacians [15, 11]. These operators are the main ingredients of filtering techniques based on variational principles, but few of them have been considered for directed graphs. We have recently proposed several formulations of the $p$-Laplacian [11]. In this paper we describe the unnormalized case used by the filtering process presented in the next section.

Given a data F, its gradient over an weighted graph $\mathrm{W}$ can be defined as $\nabla^{\mathrm{W}} \mathrm{F}=\left(\partial_{j}^{\mathrm{W}} \mathrm{F}\right)_{j \in N_{v}}$, with $\partial_{j}^{\mathrm{W}} \mathrm{F}=$ $\left[\sqrt{w_{i, j}}\left(f_{j, c}-f_{i, c}\right)\right]_{i \in N_{v}, c \in N_{c}}$ the directional differences according to vertex $j$. The gradient at a vertex $i$ is thus given by $\nabla_{i}^{\mathrm{W}} \mathrm{F}=\left[\sqrt{w_{i, j}}\left(f_{j, c}-f_{i, c}\right)\right]_{j \in N_{v}, c \in N_{c}}$. Its magnitude

$$
\begin{aligned}
\left|\nabla_{i}^{\mathrm{W}} \mathrm{F}\right| & =\left(\sum_{j=1}^{n_{v}} w_{i, j} \sum_{c=1}^{n_{c}}\left(f_{j, c}-f_{i, c}\right)^{2}\right)^{1 / 2} \\
& =\left(\sum_{j=1}^{n_{v}} w_{i, j}\left\|\mathrm{f}_{j}-\mathrm{f}_{i}\right\|^{2}\right)^{1 / 2}=\left(\sum_{c=1}^{n_{c}}\left|\nabla_{i}^{\mathrm{W}} \mathrm{F}_{c}\right|^{2}\right)^{1 / 2}
\end{aligned}
$$

with $\|\mathrm{f}\|=\sqrt{\sum_{c=1}^{n_{c}} f_{c}^{2}}$ and $\mathrm{F}_{c} \in \mathbb{R}^{n_{v}}$ the restriction of $\mathrm{F}$ to the $c$-th component, provides a basic tool for measuring the variations of $F$ at each vertex. The regularity of $F$ over the graph can then be measured by its $p$-total variation ( $p \mathrm{TV}$ ) defined as the $L_{1}$ norm of a power of the gradient magnitude:

$$
\|\mathrm{F}\|_{p \mathrm{TV}}=\left\|\left|\nabla^{\mathrm{W}} \mathrm{F}\right|^{p}\right\|_{1}=\sum_{i=1}^{n_{v}}\left|\nabla_{i}^{\mathrm{W}} \mathrm{F}\right|^{p}
$$

where $p \in[1,+\infty)$ controls the degree of regularity, and $\left|\nabla^{\mathrm{W}} \mathrm{F}\right|=\left[\left|\nabla_{i}^{\mathrm{W}} \mathrm{F}\right|\right]_{i \in N_{v}}$. As for undirected graphs [16, 17], it is easy to show that $\nabla\|\mathrm{F}\|_{p \mathrm{TV}}=p \mathrm{~L}_{p, \mathrm{~F}} \mathrm{~F}$ holds, with

$$
\mathrm{L}_{p, \mathrm{~F}}=\operatorname{diag}\left(\left(\mathrm{W}_{p, \mathrm{~F}}+\mathrm{W}_{p, \mathrm{~F}}^{T}\right) 1_{n_{v}}\right)-\left(\mathrm{W}_{p, \mathrm{~F}}+\mathrm{W}_{p, \mathrm{~F}}^{T}\right)
$$

and $\mathrm{W}_{p, \mathrm{~F}}=\operatorname{diag}\left(\left|\nabla^{\mathrm{W}} \mathrm{F}\right|^{p-2}\right) \mathrm{W}$. In other terms, we have:

$$
\left[\mathrm{L}_{p, \mathrm{~F}} \mathrm{~F}\right]_{i, c}=\sum_{j=1}^{n_{v}}\left(\frac{w_{i, j}}{\left|\nabla_{i}^{\mathrm{W}} \mathrm{F}\right|^{2-p}}+\frac{w_{j, i}}{\left|\nabla_{j}^{\mathrm{W}} \mathrm{F}\right|^{2-p}}\right)\left(f_{i, c}-f_{j, c}\right)
$$

This defines the $p$-Laplacian of $\mathrm{F}$ on $\mathrm{W}$. The Laplacian $\mathrm{L}$ is retrieved for $p=2$, and for $p=1, \mathrm{~L}_{1, \mathrm{~F}} \mathrm{~F}$ defines the weighted (mean) curvature of $\mathrm{F}$. The $p$-Laplacian is non-linear, and $\mathrm{L}_{p, \mathrm{~F}}$ is symmetric, positive semi-definite, and can be viewed as a data-dependent Laplacian. It can be rewritten as $\mathrm{L}_{p, \mathrm{~F}}=$ $\operatorname{diag}\left(\overline{\mathrm{W}}_{p, \mathrm{~F}} 1_{n_{v}}\right)-\overline{\mathrm{W}}_{p, \mathrm{~F}}$ with $\overline{\mathrm{W}}_{p, \mathrm{~F}}=\mathrm{W}_{p, \mathrm{~F}}+\mathrm{W}_{p, \mathrm{~F}}^{T}$, equivalent to a data-dependent Laplacian on an undirected graph.

The regularity measured by the $p \mathrm{TV}$ norm and the $p$ Laplacian depends on the global parameter $p$. As proposed in the following section, more flexibility can be obtained by adapting this parameter locally at each vertex.

\section{ADAPTIVE P-LAPLACIAN STRUCTURE-PRESERVING FILTERING}

We present a structure-preserving smoothing filter based on an adaptive $p$-Laplacian and an adaptive preservation of gradient magnitudes. This is inspired by a recent work in image processing [18] which considers other measures of local variations and a different formulation of their local adaptation.

\subsection{Energy formulation}

Given a data $\mathrm{F}^{0} \in \mathbb{R}^{n_{v} \times n_{c}}$, the proposed method consists to find a smoother version that minimizes an objective function $E\left(\mathrm{~F}, \mathrm{~F}^{0}\right)=\lambda_{d} E_{d}\left(\mathrm{~F}, \mathrm{~F}^{0}\right)+\lambda_{s} E_{s}\left(\mathrm{~F}, \mathrm{~F}^{0}\right)+\lambda_{r} E_{r}(\mathrm{~F})$, with $E_{d}$ a data term, $E_{r}$ a regularity term, $E_{s}$ a structure-preserving term, and $\lambda_{d}, \lambda_{s}, \lambda_{e} \in[0,+\infty)$ constant balancing weights.

The data term $E_{d}$ aims at providing a solution close to $\mathrm{F}^{0}$. This is measured according to the mean square error:

$$
E_{d}\left(\mathrm{~F}, \mathrm{~F}^{0}\right)=\left\|\mathrm{F}-\mathrm{F}^{0}\right\|^{2}
$$

with $\|\mathrm{F}\|=\sqrt{\operatorname{tr}\left(\mathrm{F}^{T} \mathrm{~F}\right)}$. The local differences $\left(f_{i, c}-f_{i, c}^{0}\right)$ should be null, or low, at vertices representing parts of the data that must be preserved. This is guided by the structurepreserving term $E_{s}$. It measures a weighted mean square error between the gradient magnitude of $\mathrm{F}$ and the one of $\mathrm{F}^{0}$ on the same weighted graph $\mathrm{S}=\left[s_{i, j}\right]_{i, j \in N_{v}}$ :

$$
E_{s}\left(\mathrm{~F}, \mathrm{~F}^{0}\right)=\frac{1}{2 n_{s}} \sum_{i=1}^{n_{v}} \alpha_{i}\left(\left|\nabla_{i}^{\mathrm{S}} \mathrm{F}\right|^{2}-\left|\nabla_{i}^{\mathrm{S}} \mathrm{F}^{0}\right|^{2}\right)^{2}
$$

with $\alpha=\left[\alpha_{i}\right]_{i \in N_{v}}, \alpha_{i} \in[0,1]$ the degree of structure preservation, and $n_{s}=n_{c} \sum_{i=1}^{n_{v}} \alpha_{i}$ a normalization term. The term $\alpha$ behaves as a mask that enforces more or less the preservation of the gradient magnitude of $\mathrm{F}^{0}$. To ensure that structures in $\mathrm{F}^{0}$ are preserved, it should be defined from an indicator or a detector of saliency, as detailed in Sec. 3.3

The local differences involved in the approximation terms $E_{d}$ and $E_{s}$ should be higher at vertices representing unimportant details. This is obtained by reducing the variations of $F^{0}$ at these vertices according to an adaptive $p \mathrm{TV}$ function:

$$
E_{r}(\mathrm{~F})=\sum_{i=1}^{n_{v}} \frac{1}{p_{i}}\left|\nabla_{i}^{\mathrm{W}} \mathrm{F}\right|^{p_{i}}
$$

where $p_{i} \in[1,2]$ fixes the degree of regularity at vertex $i$ and ensures convexity of 5 . Intuitively, it should depend on $\mathrm{F}^{0}$ 
and be inversely proportional to $\alpha_{i}$, i.e. large at vertices where the data needs to be smoothed and low where the data must be preserved. Note that the graphs $\mathrm{W}$ and $\mathrm{S}$ are different. In practice, it is more coherent and computationally effective that they are structurally equivalent, i.e. $s_{i, j}=0 \Leftrightarrow w_{i, j}=0$.

Since the terms $E_{d}, E_{s}$ and $E_{r}$ are convex functions of $\mathrm{F}$, the objective function $E$ is also convex, and several methods can be used to compute a solution. In this paper we derive a simple filter related to weighted mean filtering.

\subsection{Filtering process}

To optimize the objective function $E$ w.r.t. $\mathrm{F}$, we consider the gradient $\nabla E(\mathrm{~F})=\left[\partial E(\mathrm{~F}) / \partial f_{i, c}\right]_{i \in N_{v}, c \in N_{c}}$ and try to solve the system of nonlinear partial difference equations $\nabla E(\mathrm{~F})=0$ with

$$
\nabla E(\mathrm{~F})=2\left(\mathrm{~F}-\mathrm{F}^{0}\right)+\lambda_{r} \mathrm{~L}_{r, \mathrm{~F}} \mathrm{~F}+\frac{2}{n_{s}} \lambda_{s} \mathrm{~L}_{s, \mathrm{~F}} \mathrm{~F}
$$

where $\mathrm{L}_{r, \mathrm{~F}}$ and $\mathrm{L}_{s, \mathrm{~F}}$ are defined below.

Indeed, it is straightforward that $\nabla E_{d}(\mathrm{~F})=2\left(\mathrm{~F}-\mathrm{F}^{0}\right)$, and the gradient of the regularity term $E_{r}$ is given by $\nabla E_{r}(\mathrm{~F})=\mathrm{L}_{r, \mathrm{~F}} \mathrm{~F}$, with

$$
\left[\mathrm{L}_{r, \mathrm{~F}} \mathrm{~F}\right]_{i, c}=\sum_{j=1}^{n_{v}}\left(\frac{w_{i, j}}{\left|\nabla_{i}^{\mathrm{W}} \mathrm{F}\right|^{2-p_{i}}}+\frac{w_{j, i}}{\left|\nabla_{j}^{\mathrm{W}} \mathrm{F}\right|^{2-p_{j}}}\right)\left(f_{i, c}-f_{j, c}\right)
$$

Contrary to the $p$-Laplacian described in Sec. 2, $\mathrm{L}_{r, \mathrm{~F}}$ uses here a regularity degree $\mathrm{p}=\left[p_{i}\right]_{i \in N_{v}}$ adapted to each vertex.

Similarly, the gradient of the structure preserving term $E_{s}$ is given by $\nabla E_{s}(\mathrm{~F})=\frac{2}{n_{s}} \mathrm{~L}_{s, \mathrm{~F}} \mathrm{~F}$ with

$$
\left[\mathrm{L}_{s, \mathrm{~F}} \mathrm{~F}\right]_{i, c}=\sum_{j=1}^{n_{v}}\left(a_{i} s_{i, j}+a_{j} s_{j, i}\right)\left(f_{i, c}-f_{j, c}\right)
$$

with $a_{i}=\alpha_{i}\left(\left|\nabla_{i}^{\mathrm{S}} \mathrm{F}\right|^{2}-\left|\nabla_{i}^{\mathrm{S}} \mathrm{F}^{0}\right|^{2}\right)$.

We propose to use a linearized Gauss-Jacobi iterative method to find a solution to the system of non-linear equations. Let $t$ be an iteration step, and $\mathrm{F}^{(t)}$ be the solution at step $t$. Starting with $\mathrm{F}^{(0)}=\mathrm{F}^{0}$, and $\mathrm{g}^{0}=\left|\nabla^{\mathrm{S}} \mathrm{F}^{0}\right|^{2}$, the method iterates the following steps:

$$
\begin{array}{rlrl}
\forall i, & h_{i} & \leftarrow \mid \nabla_{i}^{\left.\mathrm{W}_{\mathrm{F}}^{(t)}\right|^{p_{i}-2}} \\
\forall i, & g_{i} & \leftarrow \alpha_{i}\left(\left|\nabla_{i}^{\mathrm{S}} \mathrm{F}^{(t)}\right|^{2}-g_{i}^{0}\right) \\
\forall i, \forall j, & \bar{w}_{i, j} & \leftarrow \frac{1}{2}\left(h_{i} w_{i, j}+h_{j} w_{j, i}\right) \\
\forall i, \forall j, \quad \bar{s}_{i, j} & \leftarrow \frac{1}{n_{s}}\left(g_{i} s_{i, j}+g_{j} s_{j, i}\right) \\
\forall i, \forall c, \quad f_{i, c}^{(t+1)} & \leftarrow \frac{\lambda_{d} f_{i, c}^{0}+\sum_{j=1}^{n_{v}}\left(\lambda_{r} \bar{w}_{i, j}+\lambda_{s} \bar{s}_{i, j}\right) f_{j, c}^{(t)}}{\lambda_{d}+\sum_{j=1}^{n_{v}} \lambda_{r} \bar{w}_{i, j}+\lambda_{s} \bar{s}_{i, j}}
\end{array}
$$

until convergence or a given number of steps is reached. An iteration computes $\mathrm{F}^{(t+1)}$, at each vertex, as a weighted mean of $\mathrm{F}^{(t)}$ in the neighborhood of the vertex in the graph $\overline{\mathrm{W}}+\overline{\mathrm{S}}$. Contrary to the family of filters based on $p \mathrm{TV}$ on undirected [16, 17] and directed graphs [11], the proposed filter adapts the regularity parameter $p$ locally and enforces the gradients to be preserved according to a structure-preserving map.

\subsection{Parameters for colored 3D point clouds}

The proposed structure-preserving filter depends on several parameters: the graphs $\mathrm{W}$ and $\mathrm{S}$, the vector of local regularity degrees $\mathrm{p}$, and the vector of local structure-preserving strengths $\alpha$. They are determined by the nature of the data and the desired filtering effect. We describe them for smoothing colored 3D point clouds. Given a set $X=\left\{x_{i}\right\}_{i=1, \ldots, n_{v}}$ of $n_{v}$ points $x_{i} \in \mathbb{R}^{3}$, and the RGB colors $\mathrm{F}^{0} \in \mathbb{R}^{n_{v} \times 3}$ associated to these points $\left(n_{c}=3\right)$, we assume a connected graph $\mathrm{S}^{0}$ has already been constructed for connecting the points. When $X$ samples the surface of a $3 \mathrm{D}$ object, $\mathrm{S}^{0}$ should be the graph induced by the edges of a mesh having $X$ as vertices. But any graph can be used as long as it is connected and represents the geometric structure of the data.

The graphs $\mathrm{S}$ and $\mathrm{W}$ are constructed by connecting each point $x_{i} \in X$ to its $k$ nearest points, within an $\alpha$-hop $\mathcal{N}_{i}^{\alpha}$ in $\mathrm{S}^{0}$, according to the dissimilarity measure $d\left(x_{i}, x_{j}\right)=$ $d_{\mathrm{EMD}}\left(H\left(\Phi_{i}^{\tau}\right), H\left(\Phi_{j}^{\tau}\right)\right)$, where $\Phi^{\tau}=\left(\Phi_{i}^{\tau}\right)_{i=1, \ldots, n_{v}}$ associates to each point $x_{i}$ a feature $\Phi_{i}^{\tau}=\left(\mathrm{f}_{j}^{0}\right)_{j \in \mathcal{N}_{i}^{\tau} \cup\{i\}}$, i.e. the colors of the points $x_{j}$ around $x_{i}$ within a $\tau$-hop $\mathcal{N}_{i}^{\tau}$ in $\mathrm{S}^{0}$. Since $\mathrm{S}^{0}$ is not assumed to be regular, features do not have the same size, and a simple $L_{2}$ norm cannot be used to compare them. The Earth Mover Distance $d_{\text {EMD }}$ [19] between the histograms $H$ of the features in the $L^{*} a^{*} b^{*}$ color space is more appropriate. The retained connections are then directly used to construct the graph S. This graph is unweighted, i.e. $s_{i, j}=1$ if $x_{j}$ is among the $k$ nearest neighbors of $x_{i}$, or 0 otherwise. The graph W has the structure of S but it is weighted according to the distances between the features defined above by a parameter-less similarity:

$$
w_{i, j}=1-\frac{d_{\mathrm{EMD}}\left(H\left(\Phi_{i}^{\tau}\right), H\left(\Phi_{j}^{\tau}\right)\right)}{\max _{\substack{l=1, \ldots, n_{v} \\ s_{i, l}=1}} d_{\mathrm{EMD}}\left(H\left(\Phi_{i}^{\tau}\right), H\left(\Phi_{l}^{\tau}\right)\right)}
$$

if $s_{i, j}=1$, and 0 else. Remark that $\mathrm{S}$ and $\mathrm{W}$ are directed.

The two other parameters $\mathrm{p}$ and $\alpha$ are defined from a common saliency map $\mathrm{m}=\left[m_{i}\right]_{i=1, \ldots, n_{v}}$ which indicates, for each point $x_{i}$, the local degree of structure in $\mathrm{F}^{0}$. This is measured by the normalized sum of distances within a $\rho$-hop in $\mathrm{S}^{0}: m_{i}=\frac{1}{\left|\mathcal{N}_{i}^{\rho}\right|} \sum_{j \in \mathcal{N}_{i}^{\rho}} d_{\mathrm{EMD}}\left(H\left(\Phi_{i}^{\rho-1}\right), H\left(\Phi_{j}^{\rho-1}\right)\right)$. The parameters are then defined by :

$$
p_{i}=1+\frac{1}{1+m_{i}^{2}}, \quad \alpha_{i}=\frac{m_{i}-\min _{j} m_{j}}{\delta_{i}\left(\max _{j} m_{j}-\min _{j} m_{j}\right)}
$$

with $\delta_{i}$ the number of edges starting at $i$ in S (outgoing degree). This enables to normalize the gradients in the objective function $E_{s}$. As discussed in the previous section, $\mathrm{p}$ and $\alpha$ defined by Eq. 9 are antagonists, one for smoothing the data and the other for preserving its main structures. 

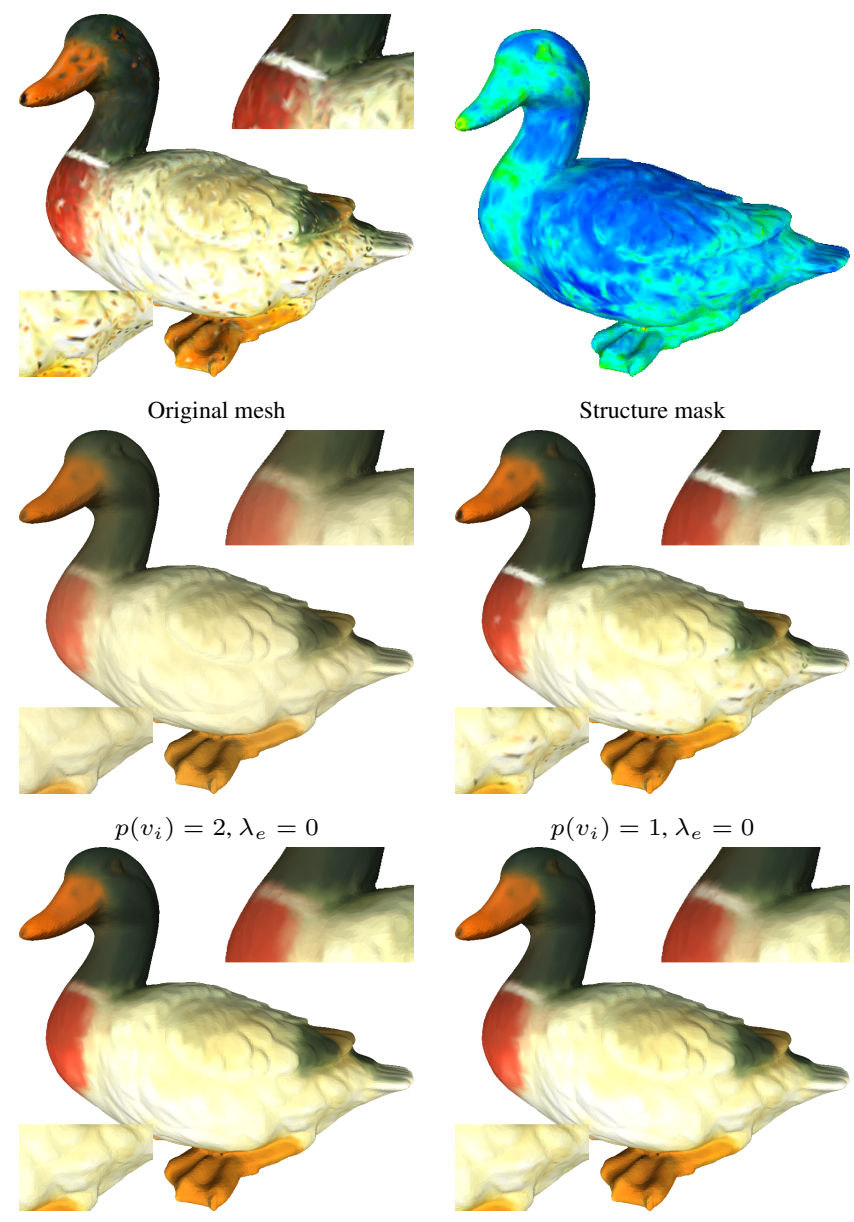

$\lambda_{e}=0$

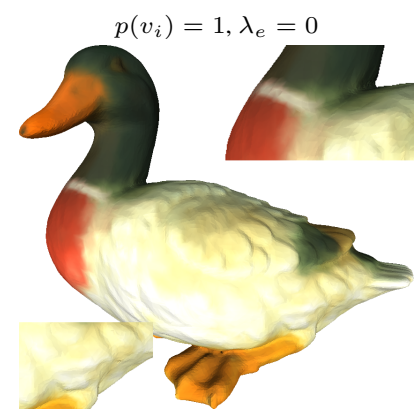

Proposed model

Fig. 1. Illustration of the proposed model.

\section{EXPERIMENTAL RESULTS AND CONCLUSION}

To illustrate the proposed feature-preserving filter, we consider colored 3D point clouds acquired by digitizing an object by means of a scanner. A mesh obtained by reconstruction techniques is also provided. We use this mesh to define the initial graph $\mathrm{S}^{0}$. For all the experiments, the parameters for constructing the graphs $\mathrm{S}$ and $\mathrm{W}$, and the saliency map $\mathrm{m}$, are fixed to $\alpha=5, \tau=1$ and $\rho=2$ for the sizes of the hops, and the number of neighbors is set to $k=10$. The balancing terms in the objective function are set to $\lambda_{d}=10^{-3}, \lambda_{r}=1$, and $\lambda_{s}=0.25$. The number of iterations is fixed to 25 .

Figure 1 shows results on a low-resolution mesh (a scan of a stuffed duck with 19247 vertices and 38490 faces) to illustrate the benefit of the proposed approach (each image shows a caption of the mesh and two cropped and zoomed areas). First row shows the original mesh and its structure mask $M$ (shown with a heat map LUT). Second row shows classical results obtained with fixed values of $p$ and no structurepreserving term. With $p=2$ (the Laplacian) a strong smoothing effect is obtained, whereas with $p=1$ (the Graph To-
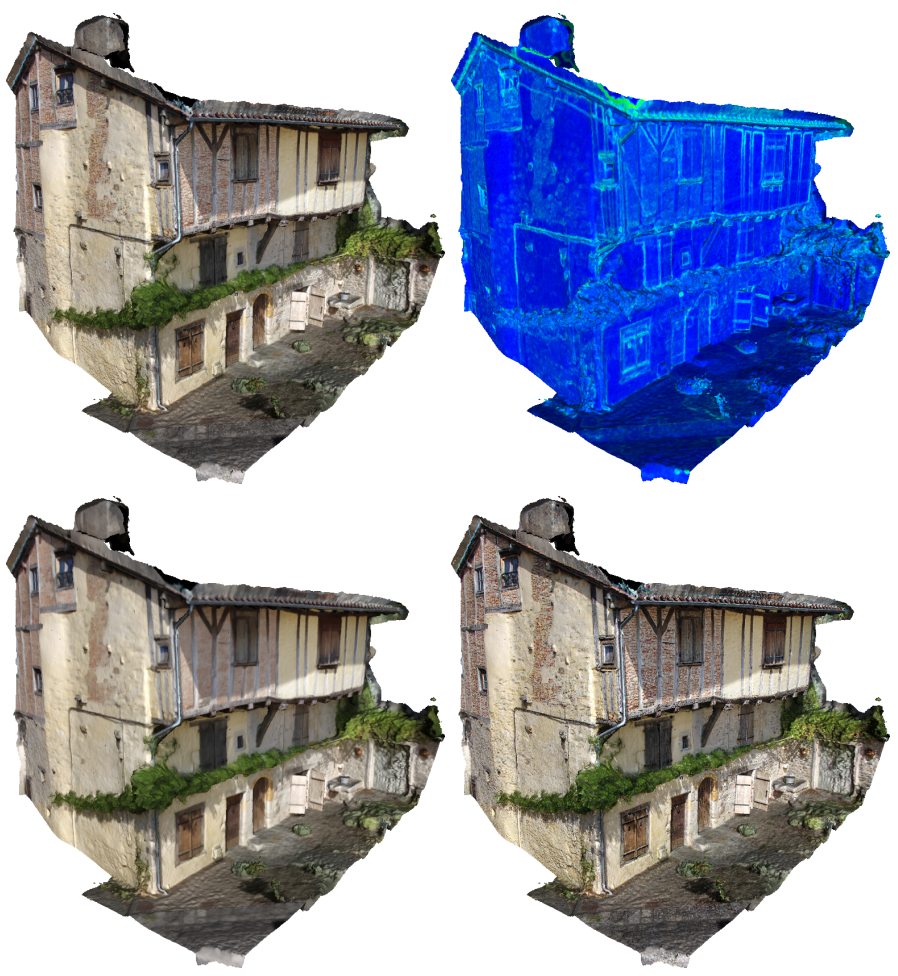

Fig. 2. First row: original mesh, structure mask. Second row: filtering and sharpening results.

tal Variation), the edges are better preserved. However some small structures are also kept. Better filtering results are obtained with a spatially varying norm and the unwanted small details are removed. However, some parts such as the duck's collar are now more blurry. This problem is then corrected by using the whole model we propose that incorporates an additional structure-preserving term. The final result is inline with our objective: to eliminate unimportant fine-scale details while maintaining the primary structures.

Figure 2 shows an exemple of 3D colored mesh editing for sharpening. The mesh is a high-resolution scan (780977 vertices and 1557701 faces) of a medieval house in the city of Bellac, France. As it can be seen, in the filtering result, the small fine details have been suppressed while preserving the strongest structures. This can be used as the base layer of a sharpness enhancement procedure [20]. The difference between the original mesh and its filtering is computed, boosted by a factor 0.8 and added back to the filtered version. As it can be seen, the sharpened result has enhanced the local contrast without artifact magnification or detail loss. Note that the enhancements are better visible at high resolution. This shows the benefit of our approach for 3D editing tasks. Further works will consider other minimization schemes as well as other editing tasks such as inpainting and abstraction. 


\section{REFERENCES}

[1] Q. Zhang, X. Shen, L. Xu, and J. Jia, "Rolling guidance filter," in European Conference on Computer Vision, 2014, pp. 815-830.

[2] H. Cho, H. Lee, H. Kang, and S. Lee, "Bilateral texture filtering," ACM Trans. Graph., vol. 33, no. 4, pp. 128:1128:8, 2014.

[3] E. S. L. Gastal and M. M. Oliveira, "Domain transform for edge-aware image and video processing," ACM Trans. Graph., vol. 30, no. 4, pp. 69, 2011.

[4] L. Xu, C. Lu, Y. Xu, and J. Jia, "Image smoothing via $L_{0}$ gradient minimization," ACM Trans. Graph., vol. 30, no. 6 , pp. 174, 2011.

[5] L. Xu, Q. Yan, Y. Xia, and J. Jia, "Structure extraction from texture via relative total variation," ACM Trans. Graph., vol. 31, no. 6, pp. 139:1-139:10, 2012.

[6] S. Fleishman, I. Drori, and D. Cohen-Or, "Bilateral mesh denoising," ACM Trans. Graph., vol. 22, no. 3, pp. 950-953, 2003.

[7] M. Kolomenkin, I. Shimshoni, and A. Tal, "Prominent field for shape processing and analysis of archaeological artifacts," International Journal of Computer Vision, vol. 94, no. 1, pp. 89-100, 2011.

[8] Z. Afrose and Y. Shen, "Mesh color sharpening," $A d$ vances in Engineering Software, vol. 91, pp. 36 - 43, 2016.

[9] O. Lézoray, "Manifold-based mathematical morphology for graph signal editing of colored images and meshes," in IEEE International Conference on Systems, Man, and Cybernetics, 2016, pp. 109-114.

[10] O. Lézoray, "3d colored mesh graph signals multi-layer morphological enhancement," in International Conference on Acoustics, Speech and Signal Processing, 2017, pp. 1358-1362.

[11] Z. Abu-Aisheh, S. Bougleux, and O. Lézoray, "plaplacian regularization of signals on directed graphs," in International Symposium on Visual Computing, 2018, vol. LNCS 11241, pp. 650-661.

[12] F. Chung, "Laplacians and the cheeger inequality for directed graphs," Annals of Combinatorics, vol. 9, no. 1, pp. 1-19, 2005.

[13] M. Hein, J.-Y. Audibert, and U. von Luxburg, "Graph laplacians and their convergence on random neighborhood graphs," Journal of Machine Learning Research, vol. 8, pp. 1325-1368, 2007.
[14] R. Olfati-Saber and R. M. Murray, "Consensus problems in networks of agents with switching topology and time-delays," IEEE Trans. Automat. Contr., vol. 49, no. 9, pp. 1520-1533, 2004.

[15] D. Zhou, B. Schölkopf, and T. Hofmann, "Semisupervised learning on directed graphs," in Advances in Neural Information Processing Systems, 2004, pp. 1633-1640.

[16] A. Elmoataz, O. Lezoray, and S. Bougleux, "Nonlocal discrete regularization on weighted graphs: A framework for image and manifold processing," IEEE Trans. Image Processing, vol. 17, no. 7, pp. 1047-1060, 2008.

[17] S. Bougleux, A. Elmoataz, and M. Melkemi, "Local and nonlocal discrete regularization on weighted graphs for image and mesh processing," International Journal of Computer Vision, vol. 84, no. 2, pp. 220-236, 2009.

[18] Q. Fan, J. Yang, D. Wipf, B. Chen, and X. Tong, "Image smoothing via unsupervised learning," ACM Trans. Graph., vol. 37, no. 6, pp. 259:1-259:14, 2018.

[19] Y. Rubner, C. Tomasi, and L. J. Guibas, “The earth mover's distance as a metric for image retrieval," International Journal of Computer Vision, vol. 40, no. 2, pp. 99-121, 2000.

[20] Z. Farbman, R. Fattal, D. Lischinski, and R. Szeliski, "Edge-preserving decompositions for multi-scale tone and detail manipulation," ACM Trans. Graph., vol. 27, no. 3, 2008. 